\title{
Glycosyl hydrolases from Bifidobacterium adolescentis DSM20083. An overview
}

\author{
Lambertus A.M. VAN DEN BROEK, Sandra W.A. HINZ, Gerrit BELDMAN, \\ Chantal H.L. DOESWIJK-VORAGEN, Jean-Paul VINCKEN, \\ Alphons G.J. VORAGEN*
}

Laboratory of Food Chemistry, Wageningen University, PO Box 8129, 6700 EV Wageningen, The Netherlands

\begin{abstract}
It is claimed that bifidobacteria have several health-promoting effects. To increase the amount of bifidobacteria in the colon the concept of probiotics and/or prebiotics can be applied. Bifidobacterium adolescentis is one of the main species of bifidobacteria in the gastro-intestinal tract of human adults. B. adolescentis is able to degrade a wide range of oligosaccharides and a number of glycosyl hydrolases have been characterized in detail. The hydrolytic activity of the glycosyl hydrolases from $B$. adolescentis toward prebiotics like arabinoxylan-oligosaccharides, isomalto-oligosaccharides, arabinogalactan, and sucrose-based oligosaccharides (raffinose, stachyose, and fructo-oligosaccharides) is reviewed. Alternatively, some of these glycosyl hydrolases are able to catalyze transglycosylation, which allows them to elongate oligosaccharides and to prepare potentially prebiotic oligosaccharides. Such oligosaccharides might be used to influence the microbial composition in the more distal parts of the colon. In nature, not all enzyme-substrate encounters are transglycosylating. So, the hydrolytic activity of the enzyme makes the oligosaccharide elongation less efficient than desired. Site-directed mutagenesis was applied to improve the transglycosylation reaction of the $\alpha$-galactosidase from $B$. adolescentis.
\end{abstract}

Bifidobacterium / prebiotic / glycosyl hydrolase / transglycosylation / site-directed mutagenesis

Résumé - Les glycosyl hydrolases de Bifidobacterium adolescentis DSM20083. Il est affirmé que les bifidobactéries ont des propriétés bénéfiques sur la santé. Pour augmenter la teneur en bifidobactéries au niveau du colon, le concept de probiotiques et/ou prébiotiques peut être appliqué. Bifidobacterium adolescentis est l'une des principales espèces de bifidobactéries dans le tractus gastro-intestinal des adultes. Certaines des glycosyl hydrolases de B. adolescentis ont été caractérisées en détail et elles semblent dégrader un large éventail d'oligosaccharides. L'activité d'hydrolyse des glycosyl hydrolases de $B$. adolescentis envers des prébiotiques tels que les arabino-xylanes, isomaltooligosaccharides, arabino-galactanes et les oligosaccharides contenant du saccharose (raffinose, stachyose et fructo-oligosaccharides) est passée en revue. De plus, certaines de ces glycosyl hydrolases peuvent être utilisées pour préparer des oligosaccharides prébiotiques potentiels par transglycosylation, ce qui permet d'allonger les oligosaccharides. De tels oligosaccharides peuvent être utilisés pour influencer la composition microbienne au niveau des parties plus distales du colon. Les produits de rencontre enzymes-substrates ne sont pas tous transglycosylés naturellement et l'activité d'hydrolyse de l'enzyme entraîne une élongation de l'oligosaccharide moindre que celle désirée. La mutagenèse dirigée a été appliquée pour améliorer la réaction de transglycosylation de l' $\alpha$-galactosidase de $B$. adolescentis.

Bifidobacterium / prébiotique / glycosyl hydrolases / transglycosylation / mutagenèse dirigée

\footnotetext{
* Corresponding authors: Fons.Voragen@wur.nl
} 


\section{INTRODUCTION}

The market for functional foods is rapidly growing, which is due to the fact that consumers are becoming more and more aware of the link between health, nutrition, and diet. Probiotics and/or prebiotics can be classified as functional food because it is claimed that they have health-promoting properties for the consumer $[6,25,27]$. A prebiotic is 'a non-digestible food ingredient that beneficially affects the host by selectively stimulating the growth and/or activity of one or a limited number of bacteria in the colon that have the potential to improve host health' [4]. It is generally accepted that mainly the growth of bacteria from the genera Lactobacillus or Bifidobacterium should be increased [28]. Examples of non-digestible oligosaccharides (NDOs), that can act as prebiotic, are fructo-oligosaccharides, $\alpha$-galacto-oligosaccharides, $\beta$-galacto-oligosaccharides, and xylo-oligosaccharides [5]. It is assumed that bifidobacteria and lactic acid bacteria contain the required glycosyl hydrolases to convert the NDOs into fermentable sugars. This is corroborated by the genome sequence of Bifidobacterium longum [24] and Lactobacillus lactis [11], which indicate that an array of glycosyl hydrolases, glycosyl transferases, carbohydrate esterases, and carbohydrate binding modules is present. Especially $B$. longum contains a high number of these genes involved in carbohydrate metabolism (i.e. $5 \%$ in B. longum and $2.5 \%$ in L. lactis) indicating that they can utilize a wide range of carbohydrates.

Enzymatic hydrolysis of glycosidic bonds can be carried out with one of two stereochemical outcomes: net retention or net inversion of the anomeric configuration $[12,26]$. Retaining glycosyl hydrolases can transfer the non-reducing moiety of a carbohydrate donor molecule to a carbohydrate acceptor molecule instead of water, which yields elongated oligosaccharides with possibly new types of linkage. Inverting glycosyl hydrolases always use water as the acceptor molecule and are thus unable to catalyze transglycosylation.

In the gastro-intestinal tract of human adults, $B$. adolescentis is one of the predo- minant groups of Bifidobacterium sp. [18, 23]. To understand the utilization of carbohydrates by $B$. adolescentis, a more detailed study of their glycosyl hydrolases is required. Therefore, in the first part of this review we have embarked on investigating the substrate specificity of glycosyl hydrolases from B. adolescentis DSM20083, in particular to understand why certain oligosaccharides can have a prebiotic function. Recently, it was found that Bifidobacterium sp. had the highest growth rate on $\beta$-galactooligosaccharides mixtures produced from lactose by its 'own' $\beta$-galactosidases [21]. This underlines the potential of using retaining enzymes from bifidobacteria for synthesis of prebiotic oligosaccharides by transglycosylation. In the second part, we report on site-directed mutagenesis to increase the transglycosylation activity of the $\alpha$-galactosidase from $B$. adolescentis.

\section{GLYCOSYL HYDROLASES FROM BIFIDOBACTERIUM ADOLESCENTIS}

Carbohydrates play an important role in the gastro-intestinal tract of humans. Besides their direct physiological effect, they also affect the gut ecosystem, which significantly contribute to the well being of humans [2]. To influence the microbiota, especially to increase the number of bifidobacteria, more knowledge is needed of what kind of oligo- and polysaccharides can be used. Identification and biochemical characterization of glycosyl hydrolases from bifidobacteria will give more insight in this. Although, there is a strong competition in the colon between the different microorganisms for the various carbon sources, and also other inhabitants may, besides bifidobacteria, proliferate that were not detected to date. In Figure 1 is a cartoon that gives an overview of some well described glycosyl hydrolases of $B$. adolescentis DSM20083 [9, 30-34, 36, 37]. Here, we discuss the possible role of these glycosyl hydrolases involved in the utilization of prebiotics by B. adolescentis. 


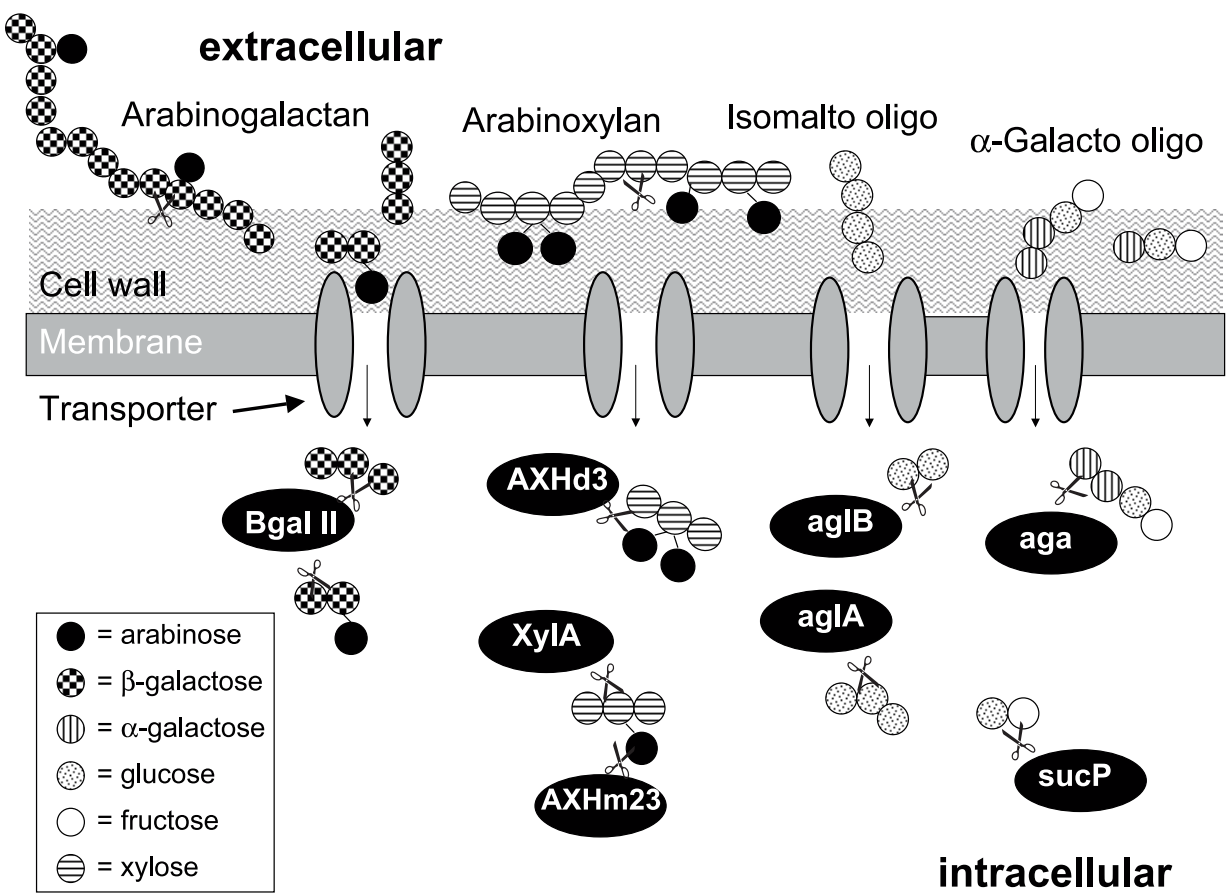

Figure 1. Overview of isolated and/or cloned glycosyl hydrolases from Bifidobacterium adolescentis DSM20083 able to utilize arabinoxylan-oligosaccharides, isomalto-oligosaccharides, $\alpha$-galactooligosaccharides, and arabinogalactan. Aga is $\alpha$-galactosidase; aglA and aglB are $\alpha$-glucosidases; sucP is sucrose phosphorylase; AXHd3 and AXHm23 are arabinofuranohydrolases d 3 and $\mathrm{m} 23$, respectively; xylA is a putative xylanase; and bgal II is $\beta$-galactosidase.

\subsection{Arabinoxylan}

It is known that arabinoxylan-oligosaccharides (XOS) can be fermented by B. longum and B. adolescentis [38]. Van Laere et al. [34, 36] characterized two different arabinofuranosidases from $B$. adolescentis able to release arabinose residues from arabinoxylan. These enzymes were named AXHd3, which hydrolyzed only C3-linked arabinosyl residues from double substituted xylosyl residues, and AXHm23, which released only arabinosyl residues that were C-2 or C-3 linked of single substituted xylosyl residues. Together with $\beta$-xylosidase these two enzymes were able to degrade XOS completely.

Recently, the AXHd3 gene was cloned and another gene preceding this sequence was also identified [33]. This gene (xylA) showed high homology with a putative endo-xylanase from Bacillus halodurans. The gene encodes for an enzyme ( $x y l A)$ that could be responsible for degradation of arabinoxylan into oligomers. However, the $x y l A$ gene did not contain a sequence for a secretion signal peptide. It remains to be determined if XylA is a true endo-xylanase or a glucanase, because this enzyme is classified into glycoside hydrolase family (GHfamily) 8 which contains mainly glucanases. The cloning and characterization of this enzyme is now in progress.

The presence of the above-mentioned enzymes shows why $B$. adolescentis is able to grow on XOS. B. longum is also able to ferment this substrate and its genome sequence revealed thirteen putative genes 
involved in arabinoxylan degradation [24]. In contrast, preliminary results of the genome sequence of $B$. breve UCC2003 showed the absence of arabinoxylan degrading enzymes [39], indicating that XOS has some selectivity within the genus Bifidobacterium.

\subsection{Isomalto-oligosaccharides}

One of the prebiotic products on the Japanese market is composed of isomaltooligosaccharides [1]. These oligosaccharides contain glucose units that are $\alpha-1,6-$ linked. Two $\alpha$-glucosidase genes have been cloned from B. adolescentis [31]. AglB (EC 3.2.1.20) was able to degrade maltose $(\alpha-$ 1,4-linkage) and isomaltose ( $\alpha-1,6-$ linkage), but not isomaltotriose. AglA (EC 3.2.1.10) showed no activity towards maltose but showed high activity toward isomaltose and isomaltotriose. AglA and not AglB could play a role in the utilization of isomalto-oligosaccharides by $B$. adolescentis.

\subsection{Sucrose-based oligosaccharides}

$\alpha$-Galacto-oligosaccharides like raffinose and stachyose are assumed to promote bifidobacterial growth in the colon [5]. $B$. adolescentis contains an $\alpha$-galactosidase $[15,30,35]$, which is able to split off the galactosyl units from these oligosaccharides, resulting in the formation of galactose and sucrose.

Sucrose can be converted by sucrose phosphorylase to D-fructose and glucose1-phosphate. This reaction is energetically advantageous, because it is an alternative for the ATP-requiring phosphorylation of glucose by hexokinase. Therefore, sucrose phosphorylase might play an important role in the fermentation pathway of sucrose obtained after hydrolysis of raffinose and stachyose. Sucrose phosphorylases genes have been cloned from B. adolescentis [32], B. lactis [29], and B. longum [10].

Another well studied prebiotic belongs to the group of fructo-oligosaccharides [5]. Some of these oligosaccharides contain a terminal sucrose unit (GF-type), such as in inulin. Bifidobacterium sp. produces a $\beta$-fructofuranosidase [20] able to split off fructosyl units from inulin, which finally results in the release of sucrose.

\subsection{Arabinogalactan}

B. adolescentis and other Bifidobacterium sp. are able to degrade arabinogalactan-oligosaccharides [38]. Recently, we have cloned the $\beta$-galactosidase gene from B. adolescentis [9], which was only induced by $\beta$-galacto-oligosaccharides with a higher degree of polymerization $(>2)$ [37]. Whereas most $\beta$-galactosidases from Bifidobacterium sp. have a preference for lactose, this enzyme showed preference for $\beta$-(1-4)-galactosides, such as in arabinogalactan-oligosaccharides derived from potato galactan. The genome sequence of B. longum reveals an endo-galactanase, which is probably membrane-anchored. We have expressed this enzyme and demonstrated that it can degrade arabinogalactan. It remains to be established whether $B$. adolescentis produces such an enzyme. It is also possible that $B$. longum produces arabinogalactan-oligosaccharides, which can be utilized by $B$. adolescentis in vivo. The presence of a $\beta$-galactosidase with preference for $\beta$-1,4-linkages together with an endo-galactanase may account for a rather complete system for the utilization of arabinogalactan in bifidobacteria.

\section{IMPROVEMENT OF THE TRANSGLYCOSYLATION ACTIVITY OF $\alpha$-GALAC- TOSIDASE FROM BIFIDO- BACTERIUM ADOLESCENTIS}

As mentioned above the use of Bifidobacterium enzymes for oligosaccharide synthesis can be advantageous. It is also discussed that elongated oligosaccharides may exert an enhanced prebiotic effect in the more distal colonic region, where most disorders of the gut are encountered [5]. Retaining glycosyl hydrolases offer the opportunity to make such longer oligosaccharides; however, there is always a competition between transglycosylation and hydrolysis. Depending on the enzyme, hydrolysis often exceeds transglycosylation. 
Experiments in our laboratory have shown that $\alpha$-galactosidase from $B$. adolescentis is a rather efficient transglycosylase in comparison with the other retaining glycosyl hydrolyses [9, 30-32, 35]. Therefore, $\alpha$-galactosidase was chosen for further improvement of its transglycosylation activity.

\subsection{Site-directed mutagenesis of $\alpha$-galactosidase from Bifidobacterium adolescentis}

The literature shows that transglycosylation can be improved by site-directed mutagenesis of the amino acids in the vicinity of the catalytic amino acids [7, 13, 17]. However, the $\alpha$-galactosidase of $B$. adolescentis belongs to GH-family 36 of which no $3 \mathrm{D}$ structure is currently available, and information on its catalytic residues is lacking. On the other hand, GH-family 36 enzymes, containing primarily $\alpha$-galactosidases from prokaryotic origin, have a strong relationship with GH-family 27 enzymes, which contain mainly $\alpha$-galactosidases from eukaryotic origin. Hart et al. [8] and Ly et al. [16] identified the catalytic nucleophile in the main $\alpha$-galactosidase from $P h a$ nerochaete chrysosporium and from green coffee bean, respectively, both belonging to GH-family 27 . The position of the catalytic nucleophile was confirmed by work from Garman and Barboczi [3], who solved the 3D structure of human $\alpha$-galactosidase $(\mathrm{GH}$ family 27). To identify the catalytic nucleophile of the $\alpha$-galactosidase from $B$. adolescentis an amino acid alignment was performed with the highly conserved consensus region of all GH-family 27 enzymes containing the catalytic nucleophile and with the corresponding conserved consensus region of all GH-family 36 enzymes. Based on the alignment, we propose that D496 in YIKWD is the catalytic nucleophile in the $\alpha$-galactosidase of B. adolescentis.

In vitro site-directed mutagenesis was applied to alter a number of amino acids of the $\alpha$-galactosidase in the vicinity of the putative catalytic nucleophile. The gene coding for $\alpha$-galactosidase from $B$. adolescentis (AF124596) [30] was amplified with $P f u$ Turbo polymerase (Stratagene) and the primers GALFOR and GALREV (Tab. I).
The primer GALFOR contained an XbaI site and the primer GALREV a HindIII site. After amplification the gene was digested with $X b a \mathrm{I}$ and HindIII and ligated into the $p$ Bluescript vector (Promega, Leiden, The Netherlands), which was previously digested with these two restriction enzymes. Sitedirected mutagenesis of the $\alpha$-galactosidase was performed according to the instructions of the supplier of the Quickchange site-directed mutagenesis kit (Stratagene, Amsterdam Zuidoost, The Netherlands). Three different mutants were created H497M, K499R, and Y500L by changing the nucleotide sequence accordingly (Tab. I). Plasmids were isolated using the High Pure PCR Product Purification Kit (Boehringer, Mannheim, Germany) and were sequenced with an automated DNA-sequencer 373 (Applied Biosystems, Nieuwerkerk a/d IJssel, The Netherlands).

In a preliminary study it was observed that the degree of transglycosylation was increased at a higher $\mathrm{pH}$. Based on these results the mutations H497M and K499R were made to influence the charge in the catalytic centre. Histidine contains a basic side-chain, whereas methionine has a neutral side-chain. The difference between lysine and arginine lies in the $\mathrm{pK}$ values of their basic side-chains, 10.5 and 12.5, respectively. The mutant Y500L was based on results of Matsui et al. [17]. They hypothesized that, in the case of $\alpha$-amylase, the aromatic ring of tyrosine was involved in the binding of oligomeric substrates through a so-called "stacking interaction", whereas the hydroxyl group of tyrosine played a role in the fixation of the catalytic water molecule. Mutation of tyrosine to a non-aromatic residue (leucine) could lead to less precise positioning of the water molecule and thereby favoring transglycosylation over hydrolysis. The different mutant $\alpha$-galactosidase genes were cloned into $E$. coli, and the obtained enzymes were tested for their hydrolytic and transglycosylation activity.

The mutant enzymes were active as judged from their activity toward $p$-nitrophenyl- $\alpha$-D-galactopyranoside [30]. Wild type and mutant enzymes, purified from the supernatant with aid of a $\mathrm{Q}$-sepharose anion exchange column (Amersham Biosciences, 
Table I. Primers used for amplification and site-directed mutagenesis of the $\alpha$-galactosidase from Bifidobacterium adolescentis DSM20083. For site-directed mutagenesis both the forward and reverse primer are indicated. Altered nucleotides are indicated in bold face type, the restrictions site containing the silent mutation ${ }^{\mathrm{a}}$ is underlined.

\begin{tabular}{|c|c|}
\hline Primer & $5^{\prime} \rightarrow 3^{\prime}$ \\
\hline GALFOR & gcgctctagagcaatgacgctcattca \\
\hline GALREV & cgcgaagctttactcagatgcggacta \\
\hline H497M & $\begin{array}{l}\text { cggcatcgattacatcaaatgggatatgaacaaatacgtcaccg } \\
\text { cggtgacgtatttgttcatatcceattgatgtaatcgatgecg }\end{array}$ \\
\hline K499R & $\begin{array}{l}\text { cggcatcgattacatcaaatgggatcacaaccgetacgtcaccg } \\
\text { cggtgacgtageggttgtgatcccatttgatgtaatcgatgccg }\end{array}$ \\
\hline Y500L & $\begin{array}{l}\text { ggatcacaacaaactcgtcaccgaagcggtgtcgecgcggaccgg } \\
\text { ccggtccgcggcgacaccgcttcggtgacgagttgttgtgatcc }\end{array}$ \\
\hline
\end{tabular}

${ }^{a}$ Silent mutations were created by changing the nucleotide sequence without changing the amino acid sequence, to check by restriction analysis (ClaI and $\mathrm{SacII}$ ) if the desired mutant was obtained.

Roosendaal, The Netherlands), were incubated with melibiose at $\mathrm{pH} 8$ and the degree of transglycosylation was determined by HPAEC [30]. Y500L and H497M mutants showed a significantly higher degree of transglycosylation, (72 and $75 \%$, respectively) than the wild type enzyme (69\%), whereas the mutant K499R showed a lower degree of transglycosylation (67\%; Fig. 2). These results showed that the transglycosylation activity could be increased, which is a first step towards a more efficient production of longer oligosaccharides. Our results indicated that subtle changes around the catalytic nucleophile could influence the likelihood of transglycosylation. However, the effect of these mutations is more difficult to explain. More research is needed to elucidate the precise mechanism by which transglycosylation can be influenced.

\section{APPLICATION OF TRANSGLYCOSYLATION PRODUCTS IN DAIRY SCIENCE}

Prebiotics can be applied to target strains naturally present in the gut that have particular health benefits [22]. Transglycosylation products can be used as prebiotic or in synbiotics to achieve this. These products are already marketed as dairy products in Europe and Japan [40]. It is also possible to use the transglycosylation activity of enzymes from probiotic microorganisms to synthesize prebiotics in food products. Lamoureux et al. [14] used mixed cultures of bifidobacteria in the preparation of yoghurts. These bifidobacteria contained $\beta$-galactosidase activities that were responsible for the extracellular production of oligosaccharides with a polymerization degree of 3 from lactose. Important prerequisites for production of elongated oligosaccharides in the product are the extracellular location of the $\beta$-galactosidases and the ability of sufficiently high concentration of donor molecules (i.e., lactose). However, most $\beta$-galactosidases are not secreted by bifidobacteria. The $\beta$-galactosidase genes from $B$. longum $\mathrm{NCC} 2705$ [24] do not have a sequence encoding a signal peptide, and only one of the three $\beta$-galactosidases from $B$. bifidum contained a signal peptide [19]. It was not investigated, which of these $\beta$-galactosidases were responsible for the transglycosylation products, although $B$. infantis showed the highest production of oligosaccharides.

In our studies we have investigated the transglycosylation activity of an $\alpha$-galactosidase from B. adolescentis. In order to produce oligosaccharides from raffinose and/ or stachyose in for example soymilk this 


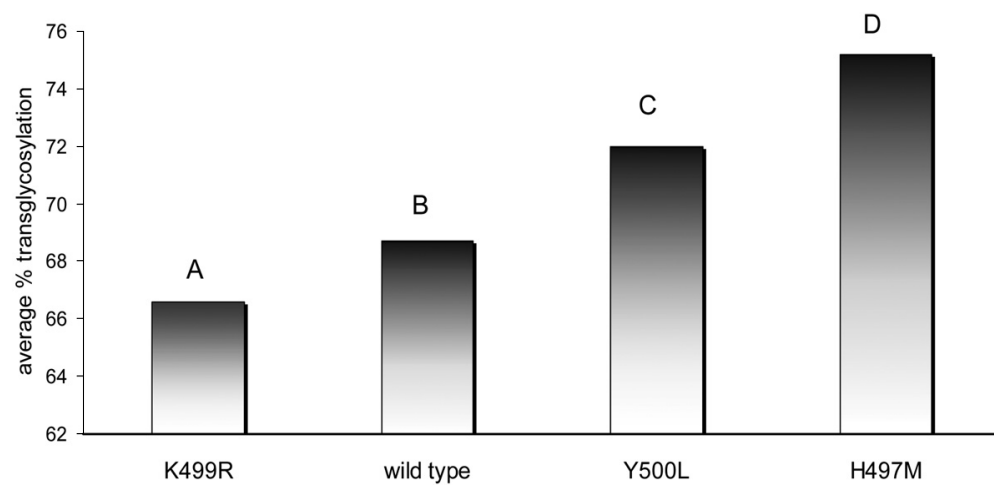

Figure 2. Average $\%$ transglycosylation activity of wild type and mutant $\alpha$-galactosidases from Bifidobacterium adolescentis DSM20083 $(n=5)$ incubated with melibiose as substrate at $\mathrm{pH} 8$ for $16 \mathrm{~h}$. A, B, C, and D represent significant difference between the mutated enzymes. Variation analysis (Oneway Anova; $P<0.05$ ) and a post-hoc test (Duncan's multiple comparison; $\alpha<0.05$ ) were used to determine significant differences between the transglycosylation activities.

enzyme should be secreted by B.adolescentis. However, this enzyme does not contain a signal peptide and the enzyme is located in the cell. The structure of the trisaccharide and tetrasaccharide synthesized from melibiose by the wild type enzyme were elucidated by NMR $(\alpha-\mathrm{D}-\mathrm{Gal} p(1,6)-\alpha-\mathrm{D}-\mathrm{Gal} p(1,6)-$ D-Glc $p$ and $\alpha-\mathrm{D}-\mathrm{Gal} p(1,6)-\alpha-\mathrm{D}-\mathrm{Gal} p(1,6)-$ $\alpha$-D-Gal $p(1,6)-\mathrm{D}-\mathrm{Gl} p$, respectively). The structure of these oligomers indicated that selective transglycosylation took place at the C6-hydroxyl group. Contrary to the example provided by Lamoureux et al. [14], these oligosaccharides should be added as ingredient to a probiotic strain to obtain a synbiotic, and are not produced in the product itself.

The genome sequence of $B$. longum revealed that most glycosyl hydrolases contain no signal peptide and are thus not secreted [24]. This makes bifidobacteria in most cases less suitable for the development of synbiotics, in which they synthesize their own prebiotics. Another drawback could be the low concentration of donor molecules in the product, which limits the synthesis of elongated oligosaccharides. Therefore, it seems more appropriate to add prebiotics to probiotic strains, instead of making them in a product. In order to reduce productions costs the transglycosylation activity of glycosyl hydrolases should be increased. Site directed mutagenesis can be one way to achieve this as indicated for the $\alpha$-galactosidase from B. adolescentis. Therefore, more research is needed to be able to influence the transglycosylation activity of glycosyl hydrolases for a higher yield. The next step is to investigate if these prebiotics and/or synbiotics have a real contribution to the well-being of humans.

Acknowledgements: A. Reijnierse is acknowledged for her contribution in the site-directed mutagenesis experiments and S. Guillotin for her help in the French translation of the abstract. This research was partly supported by the Technology Foundation STW, applied science division of NWO and the technology programme of the Ministry of Economic Affairs, as well as by the Commission of the European Communities, specific RTD progamme "Quality of Life and Management of Living Resources", QLK12000-30042.

\section{REFERENCES}

[1] Crittenden R.G., Playne M.J., Production, properties and applications of food-grade oligosaccharides, Trends Food Sci. Technol. 7 (1996) 353-361. 
[2] Falk P.G.L., Hooper T., Midvedt T., Gordon J.I., Creating and maintaining the gastrointestinal ecosystem: what we know and need to know from gnotobiology, Microbiol. Mol. Biol. Rev. 62 (1998) 1157-1170.

[3] Garman S.C., Garboczi D.N., The molecular defect leading to Fabry disease: structure of human $\alpha$-galactosidase, J. Mol. Biol. 337 (2004) 319-335.

[4] Gibson G.R., Roberfroid M.B., Dietary modulation of the human colonic microbiota: introducing the concept of prebiotics, J. Nutr. 125 (1995) 1401-1412.

[5] Gibson G.R., Ottaway P.B., Rastall R.A., Prebiotics: New developments in functional foods, Chadwick House Group Ltd, London, UK, 2000.

[6] Hammes W.P., Hertel C., Research approaches for pre- and probiotics: challenges and outlook, Food Res. Int. 35 (2002) 165-170.

[7] Hansson T., Adlercreutz P., Enhanced transglucosylation/hydrolysis ratio of mutants of Pyrococcus furiosus $\beta$-glucosidase: Effects of donor concentrations, water content, and temperature on activity and selectively in hexanol, Biotechnol. Bioeng. 75 (2000) 656665.

[8] Hart D.O., He S., Chany C.J., Withers S.G., Sims P.F.G., Sinnot M.L., Brumer H., Identification of Asp-130 as the catalytic nucleophile in the main $\alpha$-galactosidase from Phanerochaete chrysosporium, a family 27 glycosyl hydrolase, Biochemistry 39 (2000) 9826-9836

[9] Hinz S.W.A., van den Broek L.A.M., Beldman G., Vincken J.P., Voragen A.G.J., $\beta$-Galactosidase from Bifidobacterium adolescentis DSM20083 prefers $\beta(1,4)$-galactosides over lactose, Appl. Microbiol. Biotechnol. 66 (2004) 276-284

[10] Kim M., Kwon T., Lee H.J., Kim K.H., Chung D.K., Ji G.E., Byeon E.S., Lee J.H., Cloning and expression of sucrose phosphorylase from Bifidobacterium longum in $E$. coli and characterization of the recombinant enzyme, Biotechnol. Lett. 25 (2003) 12111217.

[11] Kleerebezem M., Boekhorst J., van Kranenburg R., Molenaar D., Kuipers O.P., Leer R., Tarchin R., Peters S.A., Sandbrink H.M., Fiers M.W.E.J., Stiekema W., Lankhorst R.M.K., Bron P.A., Hoffer S.M., Groot M.N.N., Kerkhoven R., de Vries M., Ursing B., de Vos W.M., Siezen R.J., Complete genome sequence of Lactobacillus plantarum WCFS1, Proc. Natl. Acad. Sci. USA 100 (2003) 1990-1995.

[12] Koshland D.E., Stereochemistry and the mechanism of enzymatic reactions, Biol. Rev. Camb. Philos. Soc. 28 (1953) 416-436.
[13] Kuriki T., Kaneko H., Yanase M., Shimada J., Handa S., Takada T., Umeyama H., Okada S., Controlling substrate preference and transglycosylation activity of neopullulanase by manipulating steric constraint and hydrophobicity in active center, J. Biol. Chem. 271 (1996) 17321-17329.

[14] Lamoureux L., Roy D., Gauthier F., Production of oligosaccharides in yoghurt containing Bifidobacteria and yoghurt cultures, J. Dairy Sci. 85 (2002) 1058-1069.

[15] Leder S., Hartmeier W., Marx S.P., $\alpha$-Galactosidase of Bifidobacterium adolescentis DSM 20083, Curr. Microbiol. 38 (1999) 101-106.

[16] Ly H.D., Howard S., Shum K., He S., Zhu A., Withers S.G., The synthesis, testing and use of 5-fluoro- $\alpha-D$-galactosyl fluoride to trap an intermediate on green coffee bean $\alpha$-galactosidase and identify the catalytic nucleophile, Carbohydr. Res. 329 (2000) 539-547.

[17] Matsui I., Shigetaka Y., Ishikawa K., Miyairi S., Fukiu S., Umeyama H., Honda K., Roles of the aromatic residues conserved in the active center of Saccharomycopsis $\alpha$-amylase for transglycosylation and hydrolysis activity, Biochemistry 33 (1994) 451-458.

[18] Matsuki T., Watanabe K., Tanaka R., Fukuda R., Oyaizu H., Distribution of bifidobacterial species in human intestinal microflora examined with 16S rRNA-gene-targeted speciesspecific primers, Appl. Environ. Microbiol. 41 (1999) 4506-4512.

[19] Møller P.L, Jørgensen F., Hansen O.C., Madsen S.M., Stougaard P., Intra- and extracellular $\beta$-galactosidases from Bifidobacterium bifidum and $B$. infantis: Molecular cloning, heterologous expression, and comparative characterization, Appl. Environ. Microbiol. 67 (2001) 2276-2283.

[20] Muramatsu K., Onodera S., Kikuchi M., Shiomi N., The production of beta-fructofuranosidase from Bifidobacterium sp., Biosci. Biotechnol. Biochem. 56 (1992) 1451-1454.

[21] Rabiu B.A., Jay A.J., Gibson G.R., Rastall R.A., Synthesis and fermentation properties of novel galacto-oligosaccharides by $\beta$-galactosidases from Bifidobacterium species, Appl. Environ. Microbiol. 67 (2001) 2526-2530.

[22] Rastall R.A., Gibson G.R., Prebiotic oligosaccharides: Evaluation of biological activities and potential future developments, in: Probiotics and prebiotics: Where are we going?, Tannock G.W.(Ed.), Caister Academic Press, Norfolk, England, 2002, pp. 107-148.

[23] Satokari R.M., Vaughan E.E., Akkermans A.D.L., Akkermans M., Saarela M., de Vos W.M., Bifidobacterial diversity in human feces detected by genus-specific PCR and denaturing gel electrophoresis, Appl. Environ. Microbiol. 67 (2001) 504-513. 
[24] Schell M.A., Karmirantzou M., Snel B., Vilanova D., Berger B., Pessi G., Zwahlen M.C., Desiere F., Bork P., Delley M., Pridmore R.D., Arigoni F., The genome sequence of Bifidobacterium longum reflects its adaptation to the human gastrointestinal tract, Proc. Natl. Acad. Sci. USA 99 (2002) 14422-14427.

[25] Shah N.P., Functional foods from probiotics and prebiotics, Food Technol. 55 (2001) 4653.

[26] Sinnott M.L., Catalytic mechanisms of enzymatic glycosyl transfer, Chem. Rev. 90 (1990) 1171-1202.

[27] Stanton C., Gardiner G., Meehan H., Collins K., Fitzgerald G., Lynch P.B., Ross R.P., Market potential for probiotics, Amer. J. Clin. Nutr. 73 (2001) 476S-483S.

[28] Tomasik P.J., Tomasik P., Probiotics and prebiotics, Cereal Chem. 80 (2003) 113-117.

[29] Trindade M.I., Abratt V.R., Reid S.J., Induction of sucrose utilization genes from Bifidobacterium lactis by sucrose and raffinose, Appl. Environ. Microbiol. 69 (2003) 24-32.

[30] Van den Broek L.A.M., Ton J., Verdoes J.C., Van Laere K.M.J., Voragen A.G.J., Beldman G., Synthesis of $\alpha$-galacto-oligosaccharides by a cloned $\alpha$-galactosidase from Bifidobacterium adolescentis, Biotechnol. Lett. 21 (1999) 441-445.

[31] Van den Broek L.A.M., Struijs K., Verdoes J.C., Beldman G., Voragen A.G.J., Cloning and characterization of two $\alpha$-glucosidases from Bifidobacterium adolescentis DSM20083, Appl. Microbiol. Biotechnol. 61 (2003) 55-60.

[32] Van den Broek L.A.M., Van Boxtel E.L., Kievit R.P., Verhoef R., Beldman G., Voragen A.G.J., Physico-chemical and transglucosylation properties of recombinant sucrose phosphorylase from Bifidobacterium adolescentis DSM20083, Appl. Microbiol. Biotechnol. 65 (2004) 219-227.

[33] Van den Broek L.A.M., Lloyd R., Beldman G., Verdoes J.C., McCleary V., Voragen A.G.J., Cloning and characterization of arabinoxylan
arabinofuranohydrolase-D3 (AXHd3) from Bifidobacterium adolescentis DSM20083, Appl. Microbiol. Biotechnol. (2005) DOI: 10.1007/s00253-004-1850-9.

[34] Van Laere K.M.J., Beldman G., Voragen A.G.J., A new arabinofuranohydrolase from Bifidobacterium adolescentis able to remove arabinosyl residues from double-substituted xylose units in arabinoxylan, Appl. Microbiol. Biotechnol. 47 (1997) 231-235.

[35] Van Laere K.M.J., Hartemink R., Beldman G., Pitson S., Dijkema C., Schols H.A., Voragen A.G.J., Transglycosidase activity of Bifidobacterium adolescentis DMS $20083 \alpha$ galactosidase, Appl. Microbiol. Biotechnol. 52 (1999) 681-688.

[36] Van Laere K.M.J., Voragen C.H.L., Kroef T., Van den Broek L.A.M., Beldman G., Voragen A.G.J., Purification and mode of action of two different arabinoxylan arabinofuranohydrolases from Bifidobacterium adolescentis DSM 20082, Appl. Microbiol. Biotechnol. 51 (1999) 606-613.

[37] Van Laere K.M.J., Abee T., Schols H.A., Beldman G., Voragen A.G.J., Characterization of a novel $\beta$-galactosidase from Bifidobacterium adolescentis DSM 20083 active towards transgalactooligosaccharides, Appl. Environ. Microbiol. 66 (2000) 1379-1384.

[38] Van Laere K.M.M., Hartemink R., Bosveld M., Schols H.A., Voragen A.G.J., Fermentation of plant cell wall derived polysaccharides and their corresponding oligosaccharides by intestinal bacteria, J. Agric. Food Chem. 48 (2000) 1644-1652.

[39] Van Sinderen D., Whole genomes of Bifidobacteria sp. Presented at the International Symposium on Propionibacteria and Bifidobacteria: Dairy and Probiotic Applications, Saint Malo, France, June 2-4, 2004.

[40] Young J., European market developments in prebiotic- and probiotic containing foodstuffs, Brit. J. Nutr. 80 (1998) 231-233. 